\title{
FOREWORD: 100 YEARS OF RELATIVITY
}

The conference "100 Years of Relativity", which was held in São Paulo, Brazil, from 22 to 24 August, 2005, was organized with a dual purpose. First, to gather researchers and students of relativity, gravitation, cosmology, field theory, astroparticle physics and astronomy, and to give them an opportunity to present their works and to attend review lectures and research seminars, as well as to provide an environment where the participants could discuss the latest breakthroughs and trends in their respective areas of interest. And second, we wanted to catch the imagination and the attention of the wider public and display the great innovations and discoveries of the last 100 years, and the excitement surrounding the breakthroughs in our understanding of the Universe. More than 200 scientists and students have participated in the conference. We held 12 plenary sessions, and the 9 parallel sessions had 51 talks and dozens of posters. Many talks presented during the conference can also be found in their original format on our website

\section{http://fma.if.usp.br/100years}

These proceedings include works that were presented during the conference in one of its nine Sections: Dark Energy, Gravitational Waves, Classical General Relativity, Observations/Experimental Cosmology, Black Holes, Semiclassical Gravity/QFT in Curved Spacetimes, Astroparticle Physics, and Quantum Gravity/Strings/Branes.

E. Abdalla (IF-USP)

L. R. Abramo (IF-USP)

G. Matsas (IFT-UNESP)

A. Saa (UNICAMP)

Guest Editors 